\title{
Die zweite Führungsebene - Brückenbauer in der Organisationsentwicklung
}

\author{
Elke Eberts
}

(C) Springer-Verlag Wien 2012

In einer aktuellen Umfrage des Galileo Instituts für Human Excellence sind die Befragten sich mehrheitlich einig: den Führungskräften der zweiten Führungsebene wird zu wenig Wertschätzung entgegengebracht. Und das ist auch in Kliniken nicht anders: Wo erfahren Leitungen im „Mittel-Management" Aufmerksamkeit jenseits der üblichen Floskeln als leidgeplagte Menschen in der Sandwichposition?

Dabei zeigen Studien, dass $80 \%$ der Veränderungsprojekte in der Umsetzung scheitern. Was macht die $20 \%$ erfolgreichen aus? Der Umsetzungserfolg steht und fällt mit einer starken mittleren Führungsebene. Wenn aber die zweite Führungsreihe für Erfolg und Misserfolg maßgeblich ist, ist dann nicht umso erstaunlicher, dass sie weitgehend ein völliges Schattendasein fristet? Ein neu entwickeltes Konzept, egal wie gut und hilfreich es ist, wird nicht mit Leben gefüllt, wenn es nicht durch Führungskräfte im Alltag getragen wird. Dazu braucht es als Führungskraft bestimmte Fähigkeiten, Erfahrung in der Mitarbeiterführung und eine ausreichende zeitliche Priorität. Weil die Aufgabe für die Funktionsfähigkeit der ganzen Organisation so wichtig ist, kommt der Auswahl der richtigen Führungskräfte im unternehmerischen Kontext tiefgreifende Bedeutung zu.
Und es zeigt sich immer wieder: direkt vorgesetzte Führungskräfte sind es, die Mitarbeiter anziehen und binden. Ihnen gelingt es oder auch nicht, Mitarbeiter in Zeiten der Veränderung für sich zu gewinnen und mitzunehmen auf dem Weg in die neue Organisation. Sehr wichtige Führungseigenschaften dabei sind Verlässlichkeit und Authentizität. Und die vielleicht wirksamste Ressource ist Führen-durchVorleben, um Mitarbeiter in der Veränderung in unsicheren Zeiten mitzunehmen.

Der Weg und das Ziel gleichzeitig, die es dauerhaft zu stabilisieren gilt, ist ein Geben und Nehmen auf Augenhöhe, das Begegnungen von Menschen ermöglicht. Den Mitarbeiter mit seinen Bedürfnissen wahrzunehmen, zu entwickeln und dafür Engagement und Verantwortung zu ernten, das ist ein hohe Kunst der mittleren Führungsebene.

Fazit: Die zweite Führungsebene trägt Erfolg und Misserfolg in der Organisationsentwicklung ganz wesentlich. Die Führungskräfte dieser Ebene sind diejenigen, die Brücken bauen - Brücken zwischen den strategischen Entscheidungen der oberen Unternehmensleitung und den Anforderungen des Tagesbetriebs und Brücken zwischen den Bedürfnissen der Vorgesetzten und Mitarbeiter.

E. Eberts $(\bowtie)$

Ruhl Consulting AG, Mannheim, Deutschland 